Brazilian Journal of Forensic Sciences, Medical Law and Bioethics

Journal homepage: www.jpebj.com.br/forensicjournal

\title{
Estimativa da Idade pelo Ângulo Mandibular Utilizando Radiografias Cefalométricas Digitais
}

\section{Age Estimate by Mandibular Angle with the Use of Digital Cephalometric Radiographs}

\author{
Flávia Pereira de França Paiva ${ }^{1}$, Adriana Paula da Costa e Silva Santiago ${ }^{2}$ \\ ${ }^{1}$ Flávia Pereira de França Paiva - Universidade Federal da Paraíba/UFPB, PB, Brasil \\ ${ }^{2}$ Adriana Paula de Andrade da Costa e Silva Santiago - Universidade Federal de \\ Pernambuco/UFPE, PE, Brasil
}

Received 7 May 2015

Resumo. A estimativa da idade possui valor indiscutível no processo de identificação humana. O ângulo mandibular tem sido apontado como um meio de estimá-la. Isto posto, o presente estudo objetivou verificar a relevância das modificações ocorridas no referido ângulo, correlacionando-as com a idade cronológica do indivíduo. Para tanto, foram utilizadas 1020 radiografias cefalométricas digitais de perfil (515 do gênero feminino e 505 do gênero masculino) de indivíduos com idades de 8 a 45 anos, obtidas por meio de Raios-X convencional. Os ângulos foram medidos através do software Radiocef Studio $2^{\circledast}$ a partir do traçado de Jarabak. Os dados foram divididos em seis grupos etários: grupo I: 08 a 10 anos, grupo II: 11 a 15 anos, grupo III: 16 a 20 anos, grupo IV: 21 a 25 anos, grupo V: 26 a 35 anos e grupo VI: 36 a 45 anos. Na análise dos dados utilizaram-se técnicas de estatística descritiva através de distribuições absolutas, percentuais e das medidas estatísticas médias e desvio padrão inferencial através dos testes $\mathrm{F}$ (ANOVA), t-Student com variâncias iguais e intervalo de confiança para a média de uma população. Para a margem de erro fixada $(5,0 \%)$ não se comprova diferenças significativas entre as idades de nenhuma das faixas etárias. As médias por faixa etária e gênero oscilaram de 125,93 a 129,91 e foram bastante aproximadas entre os gêneros sem diferenças significativas $(p>0,05)$. Conclui-se que $o$ ângulo mandibular não sofre modificações relevantes na faixa etária de 8 a 45 anos e nem em relação ao gênero.

Palavras-chave: Odontologia legal; Ângulo mandibular; Idade. 
Abstract. This research aimed at verifying the relevance of modifications occurred in the mandibular angle, correlating them with the individual's chronological age. For such, we used 1020 digital cephalometric profile radiographs of individuals whose ages ranged from 8 to 45 years old, obtained by means of conventional X-rays. The angles were measured by the Radiocef Studio $2^{\circledR}$ software as of the Jarabak's analysis. Data were divided into six age groups: group I: 08 to 10 years old, group II: 11 to 15 years old, group III: 16 to 20 years old, group IV: 21 to 25 years old, group V: 26 to 35 years old, and group VI: 36 to 45 years old. For data analysis, we used descriptive statistical techniques through absolute distributions, percentages and through average statistical measures and inferential standard deviation through the $F$ tests (ANOVA), $t$-Student with equal variances and confidence intervals for a population's average. For the fixed margin of error $(5,0 \%)$, no significant differences between ages of any age group are testified. The averages by age group and gender oscillated from 125,93 to 129,91 and were quite approximate between genders without significant differences $(p>0,05)$. It is then assumed that the mandibular angle does not suffer any relevant modifications either in the age group of 8 to 45 years old or in relation to gender.

Keywords: Legal odontology; Mandibular angle; Age.

\section{Introdução}

A identificação humana post-mortem é uma das grandes áreas de estudo e pesquisa da Medicina e Odontologia Legal. As duas ciências trabalham com o mesmo material - o corpo humano - em vários estágios do post-mortem (esquartejado, dilacerado, carbonizado, macerado, putrefeito ou esqueletizado), sempre com o mesmo objetivo: estabelecer a identidade do indivíduo. Nos processos de identificação de esqueletos, os conhecimentos de antropologia são de suma importância, pois quando são encontradas ossadas, nem sempre estão presentes todos os ossos e muitas vezes apenas o crânio é encontrado ${ }^{1}$. Dessa forma, os peritos odonto legais dispõem de vários meios que auxiliam na investigação antropológica.

A estimativa da idade do sujeito no momento da sua morte possui um valor indiscutível no processo de identificação².

Existem diversas metodologias para estimá-la: pela erupção dos dentes decíduos e permanentes, através da mineralização da cora e da raiz na dentição permanente, pela erupção do terceiro molar, pelas suturas cranianas e pelas alterações morfológicas e ângulo mandibular. A mandíbula é um dos ossos do 
corpo humano que mais sofre transformações no seu desenvolvimento e a medida do seu ângulo pode ser útil na avaliação da idade, uma vez que ele sofre variações ao longo da vida ${ }^{2-3}$. Deve-se atentar para o fato de que os cálculos para idade são estimativos, devendo utilizar a nomenclatura "estimativa" e não "determinação" da idade ${ }^{2,4-5-6}$.

Esse ângulo, formado pela linha que acompanha a borda posterior do ramo ascendente da mandíbula com a linha que acompanha a borda inferior do ramo horizontal desse osso, é obtuso no recém-nascido, diminuindo paulatinamente até a fase adulta e voltando a aumentar no idoso. Isso ocorre devido à perda dentária ${ }^{2,7-8}$. A atrofia da mandíbula por perda dos dentes é sinal de idade avançada. Na mandíbula de idosos, por vezes, há uma reabsorção alveolar total e o ângulo mandibular torna-se mais obtuso, assemelhando - se com o de um feto ${ }^{2}$. A perda precoce dos dentes e a não colocação da respectiva prótese são elementos que podem fazer com que as modificações mandibulares senis já se façam presentes antes mesmo dos 50 anos de idade ${ }^{7}$.

Todavia, sabemos que a sociedade está mais esclarecida acerca do cuidado com seus dentes; e a Odontologia, mais desenvolvida, possibilita diversos tipos de tratamentos e métodos de prevenção que amenizam a perda dentária, quando ocorre. Em razão de tudo isso, pode-se julgar que a relação outrora encontrada entre a idade e o ângulo mandibular, hoje siga caminhos diferentes, justamente pelo fato da manutenção dentária, mesmo em pessoas da terceira idade, o que não acarretaria exageradas remodelações ósseas no osso mandibular.

Um ponto crucial na Odontologia Forense é a carência, no Brasil, de pesquisas nessa área, para obtenção de padrões nacionais que sirvam de referência ao povo brasileiro ${ }^{9}$. No que diz respeito à estimativa da idade pelo ângulo da mandíbula, a literatura é pobre e raros são os estudos realizados sobre o tema. Os únicos trabalhos de referência são as tabelas de Ernestino Lopes da Silva Júnior ${ }^{3}$ e Martin apud Vanrell ${ }^{7}$.

Pensando nisso, este trabalho se propõe a relacionar a medida do ângulo mandibular com a idade e gênero de indivíduos com idade entre 8 e 45 anos utilizando para análise 1020 (mil e vinte) radiografias cefalométricas digitais de perfil (ou lateral) em face de sua nitidez e detalhamento ósseo da região submetida a exame. 


\section{Materiais e Métodos}

A pesquisa foi realizada nas dependências do Centro de Diagnóstico por Imagem (CEDRUL), localizado na cidade de João Pessoa/ PB. O universo do estudo foi composto por radiografias cefalométricas digitais, pertencentes ao banco de dados do CEDRUL. A amostra da pesquisa constou de 1020 (mil e vinte) radiografias cefalométricas digitais de perfil de indivíduos (505 do gênero masculino e 515 do feminino) com idades entre 8 e 45 anos, obtidas por meio de Raios-X convencional. Os ângulos foram medidos através do software Radiocef Studio $2 \circledast$ a partir do traçado de Jarabak. Os dados foram divididos em seis grupos etários: grupo I: 08 a 10 anos, grupo II: 11 a 15 anos, grupo III: 16 a 20 anos, grupo IV: 21 a 25 anos, grupo V: 26 a 35 anos e grupo VI: 36 a 45 anos.

Fizeram parte da pesquisa radiografias cefalométricas digitais de pacientes leucodermas, xantodermas e melanodermas cujos prontuários faziam parte do banco de dados da referida instituição radiológica. Após a análise de cada exame radiográfico, foi feito o registro dos dados de interesse da pesquisa: idade, gênero e ângulo mandibular, utilizando-se como instrumento de coleta uma ficha elaborada pelas pesquisadoras. Esta contava com espaços em branco para o preenchimento dos valores obtidos após o exame das radiografias. Dessa forma, para cada indivíduo/radiografia analisada foram preenchidos os campos referentes ao número do prontuário, gênero (masculino ou feminino), idade e ângulo mandibular, respectivamente.

$\mathrm{Na}$ análise dos dados, foram utilizadas técnicas de estatística descritiva através de distribuições absolutas, percentuais e das medidas estatísticas médias e desvio padrão inferencial através dos testes $F$ (ANOVA), t-Student com variâncias iguais e intervalo de confiança para a média de uma população. Ressalta-se que a verificação da hipótese de igualdade de variâncias foi realizada através do teste $\mathrm{F}$ de Levene. A margem de erro utilizada para a decisão dos testes estatísticos foi de 5,0\%. O programa utilizado para a digitação dos dados e obtenção dos cálculos estatísticos foi o SPSS (Statistical Package for the Social Sciences) na versão 15. 


\subsection{Considerações Éticas}

A pesquisa foi aprovada pelo Comitê de Ética em Pesquisa do Centro Universitário de João Pessoa - CEP/UNIPÊ, com base na Resolução 196/96 do Conselho Nacional de Saúde/MS.

\section{Resultados e Discussão}

Alguns estudos foram realizados a fim de verificar as modificações ocorridas no ângulo mandibular ao longo da vida.

Silva Júnior ${ }^{7}$ realizou 1.000 (mil) observações em indivíduos brancos dos dois gêneros, com idades de 5 a 45 anos, verificando que o menor ângulo encontrado mediu $105^{\circ}$ (em indivíduos de 26 a 45 anos de idade) e o maior, $135^{\circ}$ (em crianças de 5 a 10 anos de idade). Dessa forma, dentro da faixa etária analisada, o ângulo mandibular diminuiu. Honda et al. ${ }^{5}$, analisaram 240 radiografias cefalométricas de indivíduos da raça branca nas idades de 7 a 18 anos. Por meio de traçados e análise estatística verificaram que 0 ângulo não se altera em relação à idade nem ao gênero. Segundo, Martin apud Vanrell ${ }^{9}$, esse ângulo, no recém-nascido, varia de $160^{\circ}$ a $170^{\circ}$. Com a evolução etária, ele diminui paulatinamente, até atingir no adulto, entre $95^{\circ}$ e $100^{\circ}$. Após esse estágio, aumenta à razão de $0,186^{\circ}$ a cada ano, alcançando, no idoso, entre $130^{\circ}$ e $140^{\circ}$.

Os resultados obtidos no presente estudo encontram-se apresentados em forma de tabelas e gráficos.

A Tabela 1 exibe que a variabilidade expressa através do desvio padrão mostra-se reduzida desde que os valores da referida medida foram relativamente pequenos em comparação aos valores das médias e uma leve tendência de redução com a faixa etária com oscilações entre idades do mesmo período. Para a margem de erro fixada $(5,0 \%)$ não se comprova diferenças significativas entre as idades de nenhuma das faixas etárias (Figura 1). Convém salientar que a faixa etária de 36 a 45 não foi incluída nas tabelas 1 e 2 devido ao pequeno número de pacientes em algumas idades. 
Tabela 1. Média e desvio padrão da medida do ângulo mandibular por cada idade entre os pacientes do sexo masculino. ${ }^{(1)}$ Teste $F$ (ANOVA).

\begin{tabular}{|c|c|c|c|c|c|}
\hline \multirow[t]{2}{*}{$\begin{array}{l}\text { Faixa etária } \\
\text { (anos) }\end{array}$} & \multirow[t]{2}{*}{$\begin{array}{l}\text { Idade } \\
\text { (anos) }\end{array}$} & \multicolumn{2}{|c|}{ Masculino } & \multicolumn{2}{|c|}{ Intervalo de confiança com 95\% } \\
\hline & & Média & Desvio padrão & Limite inferior & Limite superior \\
\hline \multirow[t]{3}{*}{8 a 10} & 8 & 128,04 & 7,46 & 120,21 & 135,86 \\
\hline & 9 & 131,95 & 4,01 & 129,89 & 134,01 \\
\hline & 10 & 128,60 & 6,22 & 125,51 & 131,70 \\
\hline \multicolumn{2}{|c|}{ Valor de $p^{(1)}$} & $p=0,157$ & & & \\
\hline \multirow{5}{*}{11 a 15} & 11 & 129,43 & 5,60 & 126,95 & 131,92 \\
\hline & 12 & 129,38 & 6,07 & 126,81 & 131,94 \\
\hline & 13 & 127,17 & 5,96 & 124,91 & 129,44 \\
\hline & 14 & 127,87 & 6,85 & 124,75 & 130,99 \\
\hline & 15 & 125,62 & 6,73 & 122,95 & 128,28 \\
\hline \multicolumn{2}{|c|}{ Valor de $p^{(1)}$} & $p=0,166$ & & & \\
\hline \multirow[t]{5}{*}{16 a 20} & 16 & 128,14 & 6,41 & 125,79 & 130,49 \\
\hline & 17 & 127,46 & 7,51 & 124,60 & 130,31 \\
\hline & 18 & 128,76 & 7,18 & 126,21 & 131,31 \\
\hline & 19 & 127,07 & 6,84 & 124,56 & 129,58 \\
\hline & 20 & 126,83 & 8,46 & 122,14 & 131,51 \\
\hline \multicolumn{2}{|c|}{ Valor de $p^{(1)}$} & $p=0,857$ & & & \\
\hline \multirow[t]{5}{*}{21 a 25} & 21 & 125,33 & 6,51 & 122,80 & 127,85 \\
\hline & 22 & 126,46 & 7,86 & 123,14 & 129,78 \\
\hline & 23 & 125,82 & 7,15 & 122,47 & 129,17 \\
\hline & 24 & 127,60 & 7,39 & 123,92 & 131,28 \\
\hline & 25 & 126,37 & 5,14 & 122,69 & 130,04 \\
\hline \multicolumn{2}{|c|}{ Valor de $p^{(1)}$} & $p=0,870$ & & & \\
\hline \multirow[t]{10}{*}{26 a 35} & 26 & 126,79 & 6,06 & 123,12 & 130,45 \\
\hline & 27 & 126,41 & 6,69 & 121,91 & 130,90 \\
\hline & 28 & 127,03 & 4,61 & 124,37 & 129,70 \\
\hline & 29 & 123,08 & 3,19 & 115,16 & 131,00 \\
\hline & 30 & 125,89 & 5,96 & 122,10 & 129,68 \\
\hline & 31 & 127,86 & 5,72 & 124,01 & 131,70 \\
\hline & 32 & 124,62 & 5,82 & 118,52 & 130,73 \\
\hline & 33 & 125,39 & 7,64 & 118,33 & 132,46 \\
\hline & 34 & 117,94 & 6,44 & 101,94 & 133,95 \\
\hline & 35 & 123,03 & 6,90 & 105,89 & 140,16 \\
\hline \multicolumn{2}{|c|}{ Valor de $\mathrm{p}^{(1)}$} & $p=0,441$ & & & \\
\hline
\end{tabular}




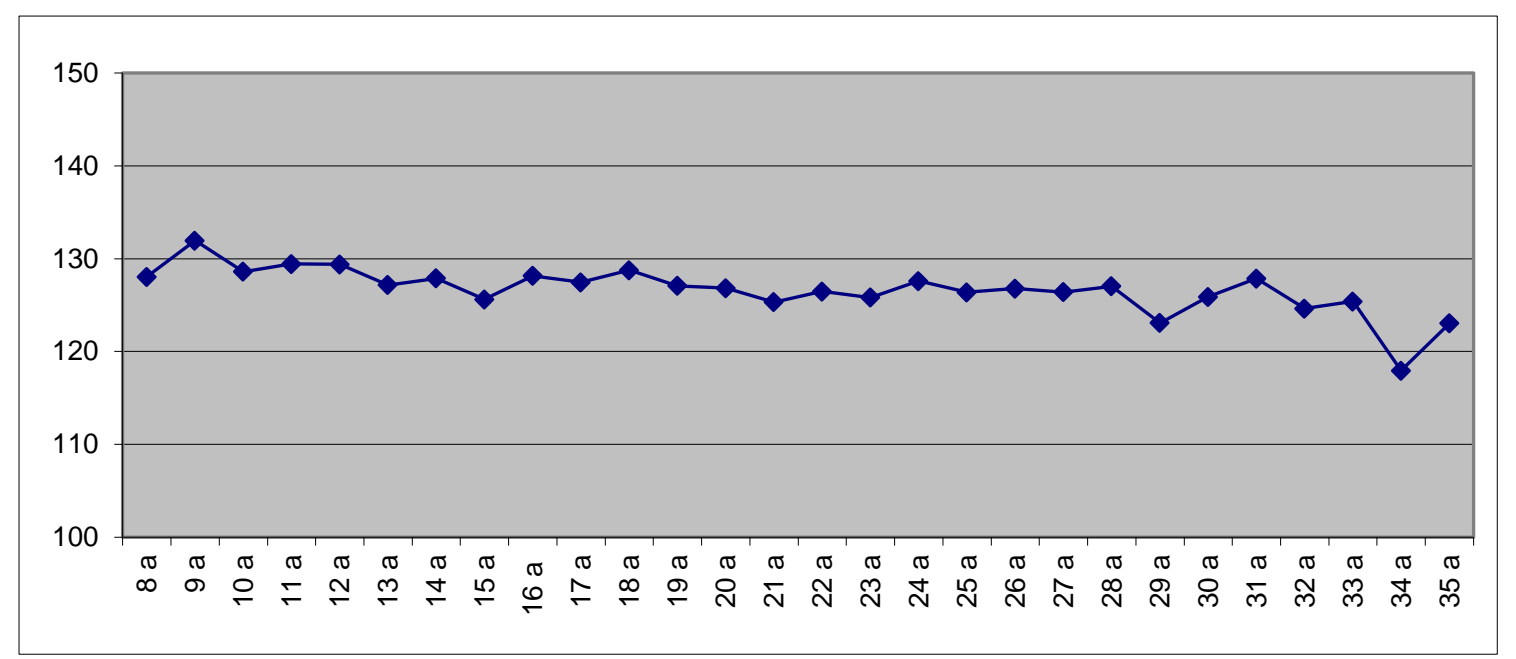

Figura 1. Média do ângulo mandibular por idade entre os pacientes do gênero masculino.

A Tabela 2 mostra que a variabilidade expressa através do desvio padrão apresenta-se reduzida desde que os valores da referida medida foram relativamente pequenos em comparação aos valores das médias e os valores oscilarem com a idade sem mostrar uma tendência. Para a margem de erro fixada $(5,0 \%)$ não se comprova diferenças significativas entre as idades de nenhuma das faixas etárias (Figura 2).

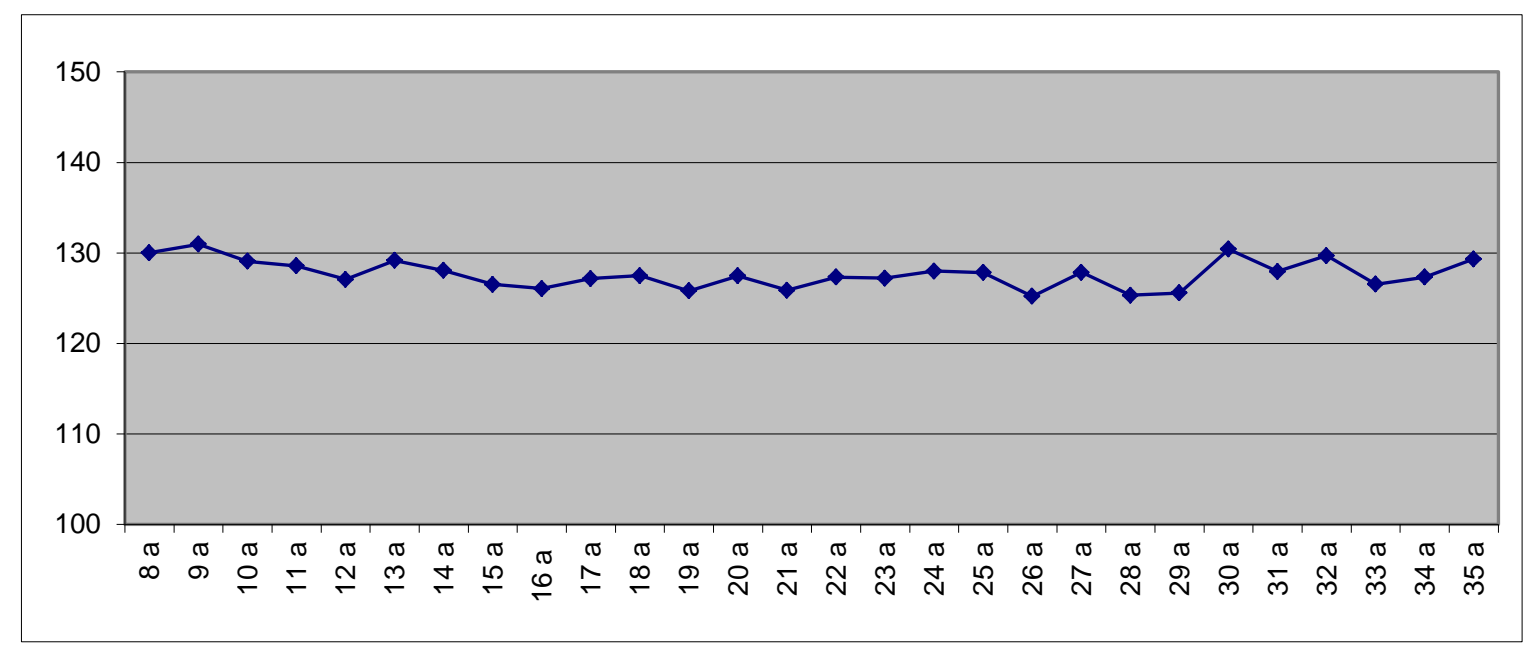

Figura 2. Média do ângulo mandibular por idade entre os pacientes do gênero feminino.

Na tabela 3 é apresentada a média e o desvio padrão do ângulo por faixa etária e gênero. Por esta tabela é possível verificar que as médias oscilaram de $125,93^{\circ}$ a $129,91^{\circ}$ e foram bastante aproximadas entre os gêneros sem diferenças significativas $(p>0,05)$. 
Tabela 2. Média e desvio padrão da medida do ângulo mandibular por cada idade entre os pacientes do gênero feminino. ${ }^{(1)}$ Teste $F$ (ANOVA).

Faixa etária

(anos) Idade (anos)

Masculino Intervalo de confiança com 95\% Média Desvio padrão

8 a 10

$\begin{array}{cc}8 & 130,02 \\ 9 & 130,96 \\ 10 & 129,07 \\ (1) & P=0,887\end{array}$

Valor de $p^{(1)} \quad P=0,887$

11 a 15

\begin{tabular}{|c|c|c|c|c|c|}
\hline \multicolumn{2}{|c|}{ Valor de $p^{(1)}$} & \multicolumn{4}{|l|}{$P=0,887$} \\
\hline \multirow[t]{5}{*}{11 a 15} & 11 & 128,57 & 5,51 & 125,38 & 131,75 \\
\hline & 12 & 127,07 & 6,61 & 124,34 & 129,80 \\
\hline & 13 & 129,16 & 5,88 & 126,79 & 131,54 \\
\hline & 14 & 128,06 & 6,61 & 125,39 & 130,73 \\
\hline & 15 & 126,52 & 6,09 & 124,20 & 128,83 \\
\hline \multicolumn{2}{|c|}{ Valor de $p^{(1)}$} & $P=0,546$ & & & \\
\hline \multirow[t]{5}{*}{16 a 20} & 16 & 126,06 & 8,65 & 122,77 & 129,35 \\
\hline & 17 & 127,17 & 6,24 & 124,84 & 129,50 \\
\hline & 18 & 127,50 & 6,21 & 124,93 & 130,06 \\
\hline & 19 & 125,80 & 5,28 & 123,62 & 127,98 \\
\hline & 20 & 127,46 & 8,62 & 123,73 & 131,18 \\
\hline \multicolumn{2}{|c|}{ Valor de $p^{(1)}$} & $P=0,858$ & & & \\
\hline \multirow[t]{5}{*}{21 a 25} & 21 & 125,87 & 7,18 & 122,84 & 128,90 \\
\hline & 22 & 127,33 & 4,25 & 125,54 & 129,12 \\
\hline & 23 & 127,21 & 5,05 & 125,07 & 129,34 \\
\hline & 24 & 127,99 & 5,35 & 125,33 & 130,65 \\
\hline & 25 & 127,84 & 7,39 & 124,57 & 131,12 \\
\hline \multicolumn{2}{|c|}{ Valor de $p^{(1)}$} & $P=0,777$ & & & \\
\hline \multirow[t]{10}{*}{26 a 35} & 26 & 125,21 & 5,05 & 122,62 & 127,81 \\
\hline & 27 & 127,86 & 11,28 & 121,05 & 134,68 \\
\hline & 28 & 125,33 & 5,57 & 121,96 & 128,69 \\
\hline & 29 & 125,58 & 7,54 & 121,03 & 130,14 \\
\hline & 30 & 130,41 & 9,06 & 120,90 & 139,92 \\
\hline & 31 & 127,94 & 7,89 & 122,64 & 133,24 \\
\hline & 32 & 129,69 & 5,84 & 125,98 & 133,41 \\
\hline & 33 & 126,54 & 4,88 & 118,78 & 134,30 \\
\hline & 34 & 127,34 & 7,77 & 117,69 & 136,98 \\
\hline & 35 & 129,33 & 7,88 & 122,74 & 135,91 \\
\hline \multicolumn{2}{|c|}{ Valor de $p^{(1)}$} & $p=0,742$ & & & \\
\hline
\end{tabular}

6,51

125,64

134,39

7,77

122,81

139,11

8,16

122,79

135,34

F. P. F. Paiva et al. 


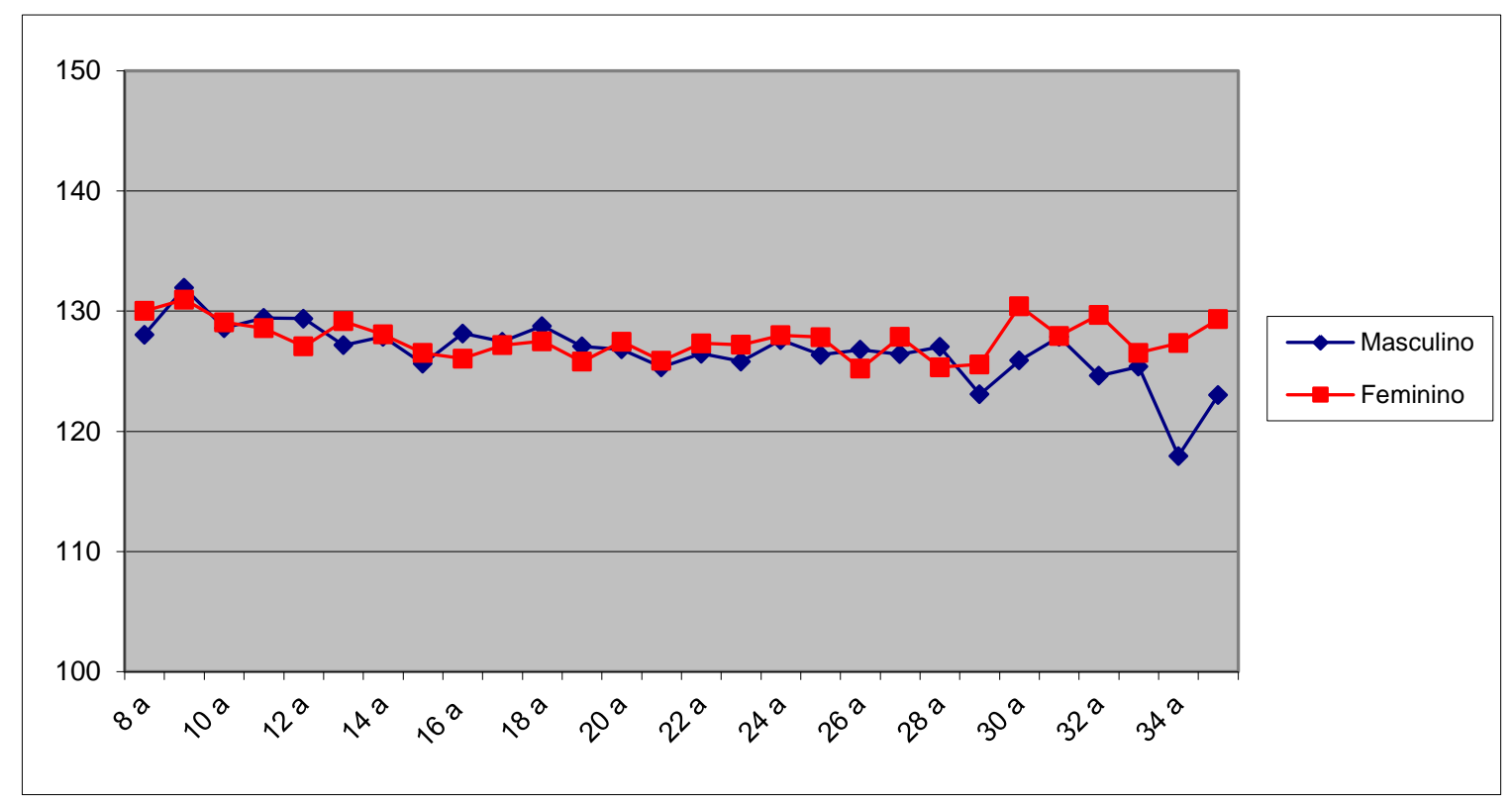

Figura 3. Média do ângulo mandibular por idade e gênero.

Tabela 3. Média e desvio padrão da medida do ângulo mandibular por faixa etária e gênero. ${ }^{(1)}$ Através do teste t-Student com variâncias iguais.

\begin{tabular}{|c|c|c|c|c|c|}
\hline \multirow{3}{*}{ Faixa etária (anos) } & \multicolumn{4}{|c|}{ Gênero } & \multirow{3}{*}{ Valor de $p$} \\
\hline & \multicolumn{2}{|c|}{ Masculino } & \multicolumn{2}{|c|}{ Feminino } & \\
\hline & Média & Desvio padrão & Média & Desvio padrão & \\
\hline 8 a 10 & 129,91 & 5,73 & 129,91 & 7,13 & $\mathrm{p}^{(1)}=1,000$ \\
\hline 11 a 15 & 127,79 & 6,32 & 127,78 & 6,19 & $\mathrm{p}^{(1)}=0,995$ \\
\hline 16 a 20 & 127,04 & 10,72 & 126,78 & 7,05 & $\mathrm{p}^{(1)}=0,812$ \\
\hline 21 a 25 & 126,21 & 6,94 & 127,20 & 5,92 & $\mathrm{p}^{(1)}=0,265$ \\
\hline 26 a 35 & 125,93 & 5,97 & 127,22 & 7,36 & $\mathrm{p}^{(1)}=0,199$ \\
\hline 36 a 45 & 129,22 & 5,76 & 129,18 & 7,25 & $\mathrm{p}^{(1)}=0,986$ \\
\hline
\end{tabular}




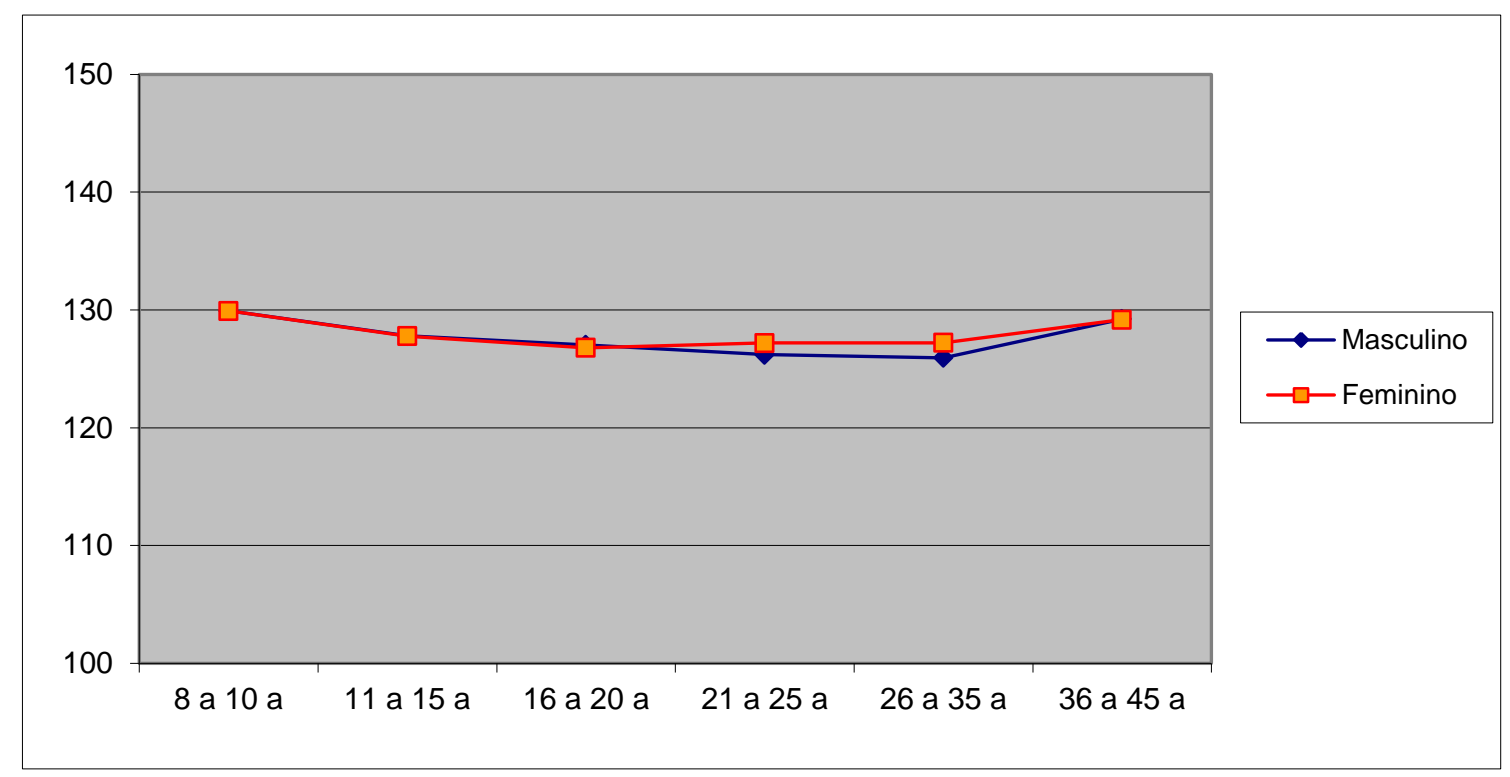

Figura 4. Média do ângulo mandibular por faixa etária e gênero.

\section{Conclusão}

Dessa forma, de acordo com a metodologia proposta neste estudo e por meio da análise dos resultados obtidos e sua discussão, pôde-se concluir que o ângulo mandibular não sofre modificações relevantes na faixa etária de 8 a 45 anos e nem em relação ao gênero.

\section{Referências}

1. Silva M. Compêndio de Odontologia Legal. 1.ed. Rio de Janeiro: Guanabara Koogan; 1997.

2. Coma JMR. Estimación de la edad en el esqueleto. In: Coma JMR. Antropología Forense. $2^{a}$ ed. Madrid: Ministerio de Justicia; 1999. p 539.

3. Silva Júnior EL. Identidade e Identificação. In: Silva Júnior EL Manual de Medicina Legal. 4aㅡ ed. São Paulo: Sugestões Literárias S.A; 1969. p 12-16.

4. Arbenz GO. Estimativa da idade. In: Arbenz GO. Medicina Legal e Antropologia Forense. São Paulo: Atheneu; 1988. p 257-258.

5. Gomez H. Identidade e Identificação. In: Gomez H. Medicina Legal. 33a ed. Rio de Janeiro: Freitas Bastos; 2004. p 45.

6. Schmidt CM. Estimativa da Idade e sua Importância Forense [Dissertação de Mestrado]. Piracicaba: Faculdade de Odontologia de Piracicaba, Universidade Estadual de Campinas, 2004.

7. Vanrell JP. Odontologia Legal e Antropologia Forense. $2^{\text {a }}$ ed. Rio de Janeiro: Guanabara Koogan; 2009. 
404 Brazilian Journal of Forensic Sciences, Medical Law and Bioethics 4(4):394-404 (2015)

8. Honda GR, Cacian DCJ, Vieira EH, Minarelli AM. Análise do ângulo da mandíbula nas idades de 7 a 18 anos, utilizando radiografias cefalométricas. Rev Odontol Unesp. 1991; v20:113-117.

9. Galvão LCC. Medicina legal. 1. ed. São Paulo: Santos; 2008. 